\title{
Circulating mRNA and microRNA profiling analysis in patients with ischemic stroke
}

\author{
SUJUAN SUN, LITAO LI, LIPENG DONG, JINMING CHENG, CONGYING ZHAO, CHU BAO and HEBO WANG
}

Department of Neurology, Hebei General Hospital, Shijiazhuang, Hebei 050050, P.R. China

Received January 15, 2019; Accepted December 3, 2019

DOI: $10.3892 / \mathrm{mmr} .2020 .11143$

\begin{abstract}
To provide insight into molecular diagnosis and individualized treatment of ischemic stroke (IS), several available datasets in IS were analyzed to identify the differentially expressed genes and microRNAs (miRNAs). Series matrix files from GSE22255 and GSE16561 (mRNA profiles), a well as GSE110993 (miRNA profile) were downloaded from the Gene Expression Omnibus database. System-level clustering was performed with GeneCluster 3.0 software, and gene annotation and pathway enrichment were performed with gene ontology analysis and Database for Annotation, Visualization and Integrated Discovery software. For a protein-protein interaction (PPI) network, Biological General Repository for Interaction Datasets and IntAct interaction information were integrated to determine the interaction of differentially expressed genes. The selected miRNA candidates were imported into the TargetScan, miRDB and miRecords databases for the prediction of target genes. The present study identified 128 upregulated and 231 downregulated genes in female stroke patients, and 604 upregulated and 337 downregulated genes in male stroke patients compared with sex- and age-matched controls. The construction of a PPI network demonstrated that male stroke patients exhibited YWHAE, CUL3 and JUN as network center nodes, and in female patients CYLD, FOS and PIK3R1 interactions were the strongest. Notably, these interactions are mainly involved in immune inflammatory response, apoptosis and other biological pathways, such as blood coagulation. Female and male upregulated genes were cross-validated with another set of Illumina HumanRef-8 v3.0 expression beadchip (GSE16561). Functional item association networks, gene function networks and transcriptional regulatory networks were successfully constructed, and the relationships between miRNAs and target genes were successfully predicted. The present study identified a number of transcription factors, including DEFA1, PDK4,
\end{abstract}

Correspondence to: Professor Hebo Wang, Department of Neurology, Hebei General Hospital, 348 Hepingxi Road, Shijiazhuang, Hebei 050050, P.R. China

E-mail: wanghbhope@hebmu.edu.cn

Key words: bioinformatics, gene, microRNA, signaling pathway, stroke
SDPR, TCN1 and MMP9, and miRNAs, including miRNA (miR)-21, miR-143/145, miR-125-5p and miR-122, which may serve important roles in the development of cerebral stroke and may be important molecular indicators for the treatment of IS.

\section{Introduction}

Ischemic stroke (IS), which is a major cause of disability and mortality worldwide, is a cerebrovascular disease caused by cerebral blood supply disorder or hemorrhage and results in the formation of an infarction zone with tissue necrosis and softening $(1,2)$. It can lead to the severe impairment of sensory and motor dysfunction, neurodegenerative diseases and sudden mortality (3). Cerebral infarction not only seriously affects the life quality of patients, but also imposes a huge burden and pressure on public health care and the social economy (4). A previous study reported that the impact caused by IS will become more apparent in the next two decades as the world's population ages and lifestyles change, especially in developing countries (5). An epidemiological study has showed that $>70 \%$ of patients with IS currently have mental and physical functional disorders (6). Tissue plasminogen activator is the only thrombolytic drug approved by the US Food and Drug Administration for the treatment of acute cerebral infarction, but its clinical application is limited by the timing of stenosis (onset) and side effects such as bleeding (7,8). A number of other previous studies have reported that transient cerebral ischemia/hypoxia can lead to neuronal death, but the mechanism is not fully understood $(9,10)$. Therefore, further understanding of the pathogenesis of cerebral infarction, finding biomarkers that can be used to diagnose and predict prognosis, and eliciting new therapeutic targets have become a significant and urgent task.

MicroRNAs (miRNAs) are highly conserved, endogenous non-coding single-stranded small RNAs that are 22-24 nucleotides long and can bind to target mRNAs with a complementary nucleic acid sequence. In general, miRNAs act post-transcriptionally to inhibit the translation of the target mRNAs or degrade the target mRNA (11). Each miRNA can regulate the expression of multiple target genes, and the same gene can also be regulated by several different miRNAs (12). Currently, >2,000 human miRNAs have been identified, and it is hypothesized that these small molecules regulate about one-third of human genes (13). Therefore, miRNA changes can lead to various diseases. A number of studies have demonstrated that miRNAs serve important biological roles in cell 
growth, proliferation, differentiation and apoptosis, as well as reconstruction of hematopoietic and damaged tissues $(14,15)$. Therefore, the role of miRNAs in neurological diseases has attracted increasing attention. In the nervous system, miRNAs are closely related to the growth and differentiation of neurons, memory and learning functions, as well as neurological degenerative diseases and mental disorders $(16,17)$. In addition, abnormal miRNA expression is detected in brain tumors, Parkinson's disease, Alzheimer's disease and IS (18-20).

Studies on the identification of target genes for miRNAs associated with cerebral infarction suggest that miRNAs play a key role in the development and progression of cerebral infarction $(21,22)$. Among them, apoptosis is the central link of this process, and related studies have indicated that apoptosis is a highly regulated and controlled death process induced by miRNAs (23). In the present study, bioinformatics analysis was performed to investigate the putative genes and miRNAs involved in the pathogenesis and disease process of IS, and the mechanism was studied at the molecular level, which provided a reference for molecular diagnosis and individualized treatment of IS.

\section{Materials and methods}

Microarray data. Two mRNA microarray datasets, GSE22255 and GSE16561, and one miRNA profile, GSE110993, of IS were obtained from the Gene Expression Omnibus (GEO) database (https://www.ncbi.nlm.nih.gov/geo). GSE22255 is based on the GPL570 (HG-U133_Plus_2) Affymetrix Human Genome U133 Plus 2.0 Array. Gene expression profiling was performed on peripheral blood mononuclear cells (PBMCs) of 20 patients with IS (10 male; 10 female) and 20 sex- and age-matched healthy control (CTL) patients using Affymetrix microarrays. GSE16561 contained a total of 63 samples based on the platform of GPL6883 Illumina HumanRef-8 v3.0 expression beadchip, in which peripheral blood samples were collected in Paxgene Blood RNA tubes from patients who were $>18$ years of age with MRI diagnosed IS $(n=39 ; 22$ females and 17 males) and CTL ( $\mathrm{n}=24 ; 14$ females and 10 males) who were non-stroke neurologically healthy. As for GSE110993, RNA sequencing results were analyzed to study expression changes of circulating miRNAs in a discovery sample of 20 patients (10 males and 10 females) with IS and 20 sex- and age-matched healthy control (CTLs) patients was further applied in independent samples for validation (40 patients with IS and 40 CTL patients) and replication (200 patients with IS, 100 CTLs).

Identification of differentially expressed genes (DEGs). Background correction and quartile data normalization of the downloaded data were performed using the robust multi-array average algorithm (24). Principal component analysis was subsequently conducted to reduce the dimension of multivariate data to two or three principal components, which can be visualized graphically with the least information loss. We used $\mathrm{R}$ software to calculate and visualize the principal component analysis (25). Probes without a corresponding gene symbol were then filtered and the average value of gene symbols with multiple probes was calculated using $\mathrm{R}$ software (26). DEGs between two groups were screened by Student's t-test and fold-change (FC) filtering with R software limma package (27). With a threshold of $\mathrm{P}<0.05$ and $\mid \mathrm{FCl}>2$, volcano plots were constructed to identify the DEGs with statistical significance between two groups by R software ggplot 2 package (data not shown) (28). In addition, hierarchical clustering and combined analyses were performed for the DEGs. The GSE16561 dataset was used to validate top 20 of DEGs in the GSE22255 dataset to identify overlapping DEGs in two microarrays.

Protein-Protein Interaction (PPI) network construction and analysis. The PPI network was created by integrating Biological General Repository for Interaction Datasets (BioGRID) and IntAct databases at EMBL-EBI. First, the PPI pairs were used to construct the PPI network. Second, the regulatory relationship between genes was visualized by using Cytoscape (version 3.4.0) (29), and analyzed through a topological property of computing network including the degree distribution of network by using the CentiScape app version $2.2(30)$.

Functional enrichment analysis. The Database for Annotation, Visualization and Integrated Discovery (DAVID) Bioinformatics resource (http://david.abcc.ncifcrf.gov) was used to conduct functional enrichment analysis to understand the underlying biological processes and pathways of aberrantly expressed intersecting genes.

Gene Ontology (GO) terms for the biological process (BP) were enriched. The most enriched term within a cluster was chosen as the one to represent the cluster. Only terms with $\mathrm{P}<0.05$ and the concentrated gene numbers $\geq 1.5$ were considered meaningful ( $\geq 1$ gene number exists).

Prediction of miRNA targets. The target genes for differentially expressed (DE) miRNAs in GSE110993 were predicted using three established programs: TargetScan version 7.2.0 (31), miRTarBase version 7.0 (32) and miRDB version 5.03 (33), which are the most popular databases of experimentally validated miRNA interactions. The genes that were predicted by all three programs were chosen as the targets of DE miRNAs and the miRNA-gene network was constructed using miRWalk 2.0 (34). Cytoscape (https://cytoscape.org; version 3.40) was used to visualize the miRNA regulatory network.

\section{Results}

Identification of differentially expressed genes in female and male IS patients. A total of 10 female and 10 male patients with IS, and 20 sex- and age-matched respective CTLs from the data in the GEO database (GSE22255) were clustered and divided into four groups (Fig. 1A): i) PBMCs_IS_M group, ii) PBMCs_IS_F group, iii) PBMCs_CTL_M group and iv) PBMCs_CTL_F group, as depicted in Fig. 1A. Principle component analysis of genes in patients with IS and CTL is presented in Fig. 1B. Following data preprocessing, the unique genes in the four groups of samples were analyzed and it was determined that a total of 1,300 genes were differentially expressed in the IS samples compared with the CTL samples. Among these DEGs, 128 genes were upregulated in females, 603 genes were upregulated in males, 232 were genes downregulated in females and 337 genes were downregulated in 
A

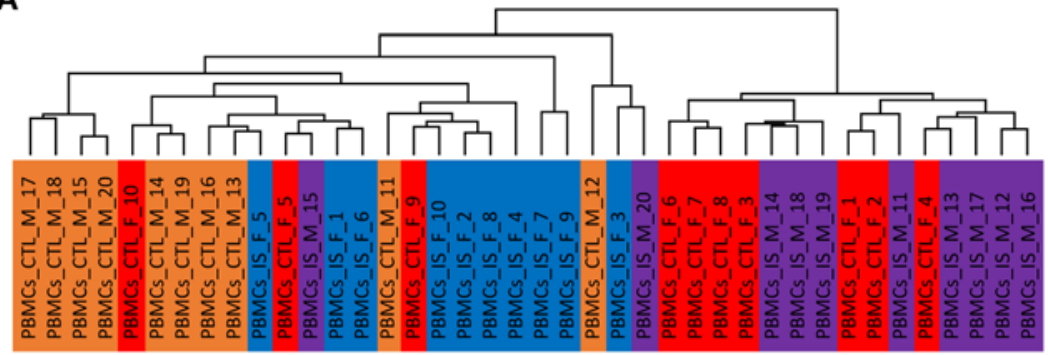

B

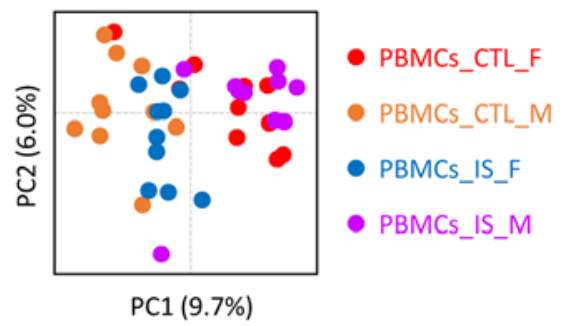

C

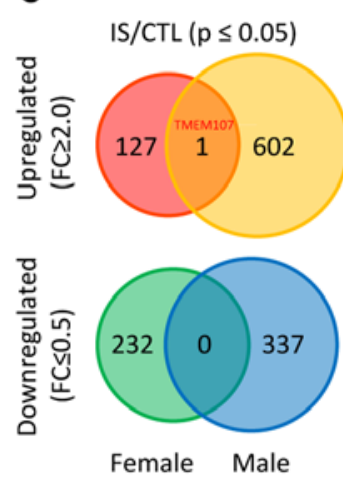

D

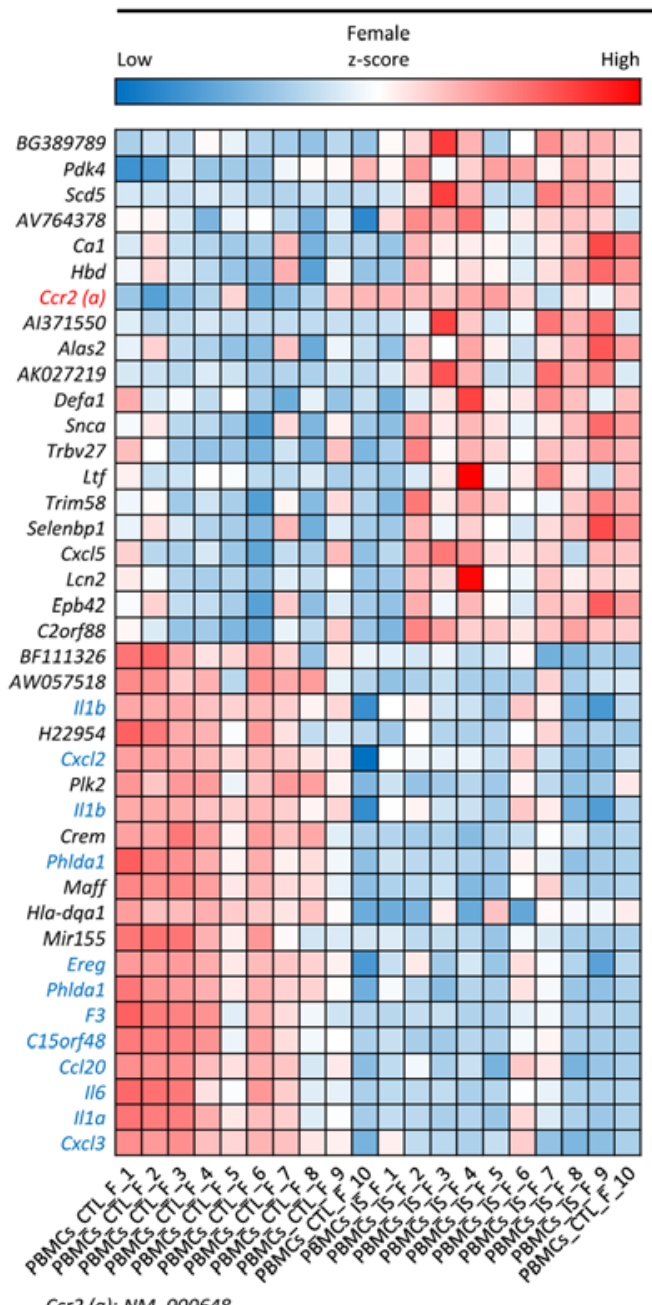

Top 20 DEGs

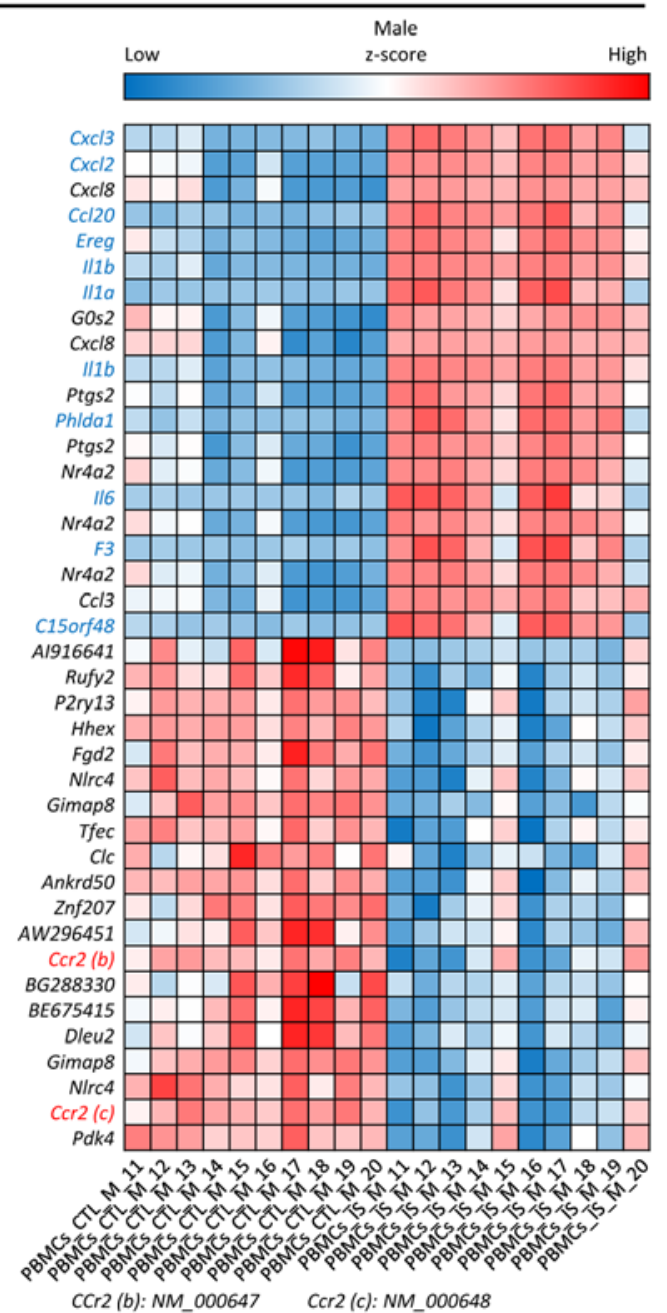

Figure 1. Identification of DEGs in female and male patients with IS. (A) Bidirectional hierarchical clustering and (B) principal component analysis of samples based on differentially expressed mRNAs. (C) Venn diagram of differentially expressed mRNAs from IS/CTL group. (D) Heatmap showing the expression levels of the top 20 mRNAs in the female (left) and male (right) dataset. The expression levels are from blue (low expression) to red (high expression). CTL, control; DEG, differentially expressed genes; F, female; FC, fold-change; IS, ischemic stroke; M, male; PBMC, peripheral blood mononuclear cells.

males. For instance, it was found that the chemokines C-X-C motif chemokine (CXCL)3, CXCL2 and CXCL8 were significantly upregulated in male IS patients. However, TMEM107 was the only overlapped upregulated DEG between the female and male group, which is displayed in a Venn diagram (Fig. 1C). A heat map of the top 20 up- and downregulated DEGs in male and female groups is shown in Fig. 1D.

Functional and pathway enrichment analyses. DAVID was used to perform GO term enrichment analyses to explore the function and pathways of 1,300 identified DEGs (Fig. 2).
GO-BP term enrichment analysis demonstrated that the upregulated genes were significantly enriched in 'blood coagulation' and 'inflammatory response' in the female IS compared with the CTL group. In addition, "positive regulation of transcription from RNA polymerase II promoter' and 'neutrophil chemotaxis' were enriched in the male IS compared with the CTL group (Fig. 2A). Downregulated genes were mainly involved in 'positive regulation of transcription' and 'immune response' in the female IS group (Fig. 2B). It was also revealed that most of the downregulated genes were involved in 'inflammatory response' and 'protein ubiquitination' in the male IS group, 
A

Female IS/CTL up top 10, FC $\geq 2.0$

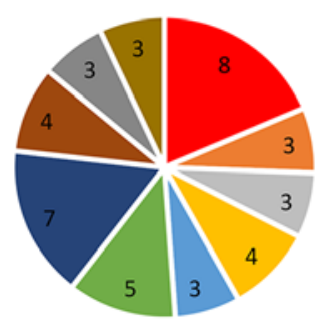

- Blood coagulation

= G-protein coupled receptor signaling pathway

= Oxygen transport

" Negative regulation of angiogenesis

- Innate immune response in mucosa

" Response to lipopolysaccharide

- Inflammatory response

- Platelet degranulation

" Erythrocyte differentiation

- Cellular iron ion homeostasis

\section{B}

Male IS/CTL up top $10, \mathrm{FC} \geq 2.0$

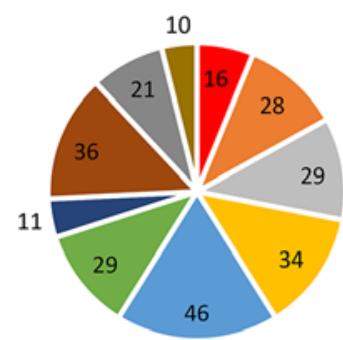

- Cellular response to lipopolysaccharide

= Inflammatory response

= Immune response

- Apoptotic process

- Positive regulation of transcription from RNA polymerase II promoter

- Negative regulation of apoptotic process

- Neutrophil chemotaxis

- Negative regulation of transcription from RNA polymerase II promoter

- Positive regulation of apoptotic process

- Positive regulation of smooth muscle cell proliferation
Female IS/CTL down top $10, \mathrm{FC} \leq 0.5$

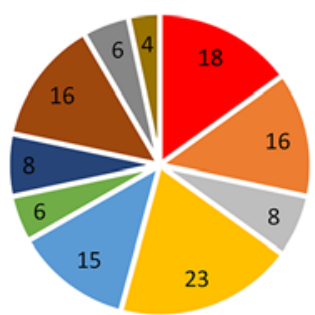

- Immune response

- Inflammatory response

= Neutrophil chemotaxis

= Positive regulation of transcription

- Negative regulation of apoptotic process

- Positive regulation of nitric oxide biosynthetic process

- Cellular response to lipopolysaccharide

- Apoptotic process

- Positive regulation of smooth muscle cell proliferation

- Positive regulation of interleukin-2 production

Male IS/CTL down top $10, \mathrm{FC} \leq 0.5$

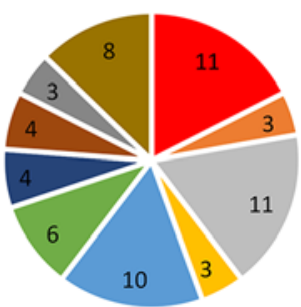

- Protein ubiquitination

= G-protein coupled purinergic nucleotide receptor signaling pathway

- Innate immune response

" Regulation of extrinsic apoptotic signaling pathway via death domain receptors

= Inflammatory response

- Positive regulation of I-kappaB kinase/NF-kappaB signaling

- Positive regulation of osteoblast differentiation

- Type I interferon signaling pathway

- Positive regulation of interleukin-8 production

= Positive regulation of apoptotic process

Figure 2. Gene Ontology biological function term enrichment analyses from IS compared with the CTL group in the (A) female and (B) male patients. The numbers in the pie slices represent the number of differentially expressed genes involved in the function. CTL, control; IS, ischemic stroke.

respectively. Of note, in both the male and female groups, the pathways of differential expression were mainly involved in 'immune response', 'inflammatory response', 'apoptotic process' and 'neutrophil chemotaxis'.

Construction of PPI network and module analysis. To investigate the associations between the DEGs and BPs, a comprehensive analysis of protein interactions of the top up- and downregulated DEGs was conducted using BioGRID, IntAct database and Cytoscape software (Fig. 3). Direct or indirect interactions were identified, and the highest-scoring nodes were screened as central genes in the female IS/CTL group, including FOS, CLU, LTF, PF4, CXCL5 and CYLD (Fig. 3). In the male IS/CTL group, the top 3 genes with the most significant expression were CUL3, YWHAE and
JUN (Fig. 4). The associations between BP and gene expression were categorized into 4 processes, namely inflammatory response, immune response, apoptotic process and neutrophil chemotaxis.

miRNA target regulatory network analysis. DE miRNAs obtained from GSE110993 database were analyzed. For the female IS/CTL group, six DE miRNAs were identified, including let-7f-5p, let-7i-5p, miR-17-5p, miR-20a-5p, miR-19b-3p and miR-205-5p (Fig. 5). In the male IS/CTL group, there were 11 DE miRNA identified, such as miR-205-5p, miR-17-5p, miR-20a-5p, miR-125a-5p, miR-92a-3p, miR-18a-5p and miR-143-3p (Fig. 6). Their target genes were predicted using TargetScan, miRTarBase and miRDB. The upregulated targets were STRADB, PDK4, SCD5, SNCA, RARA and SHBGRL2 


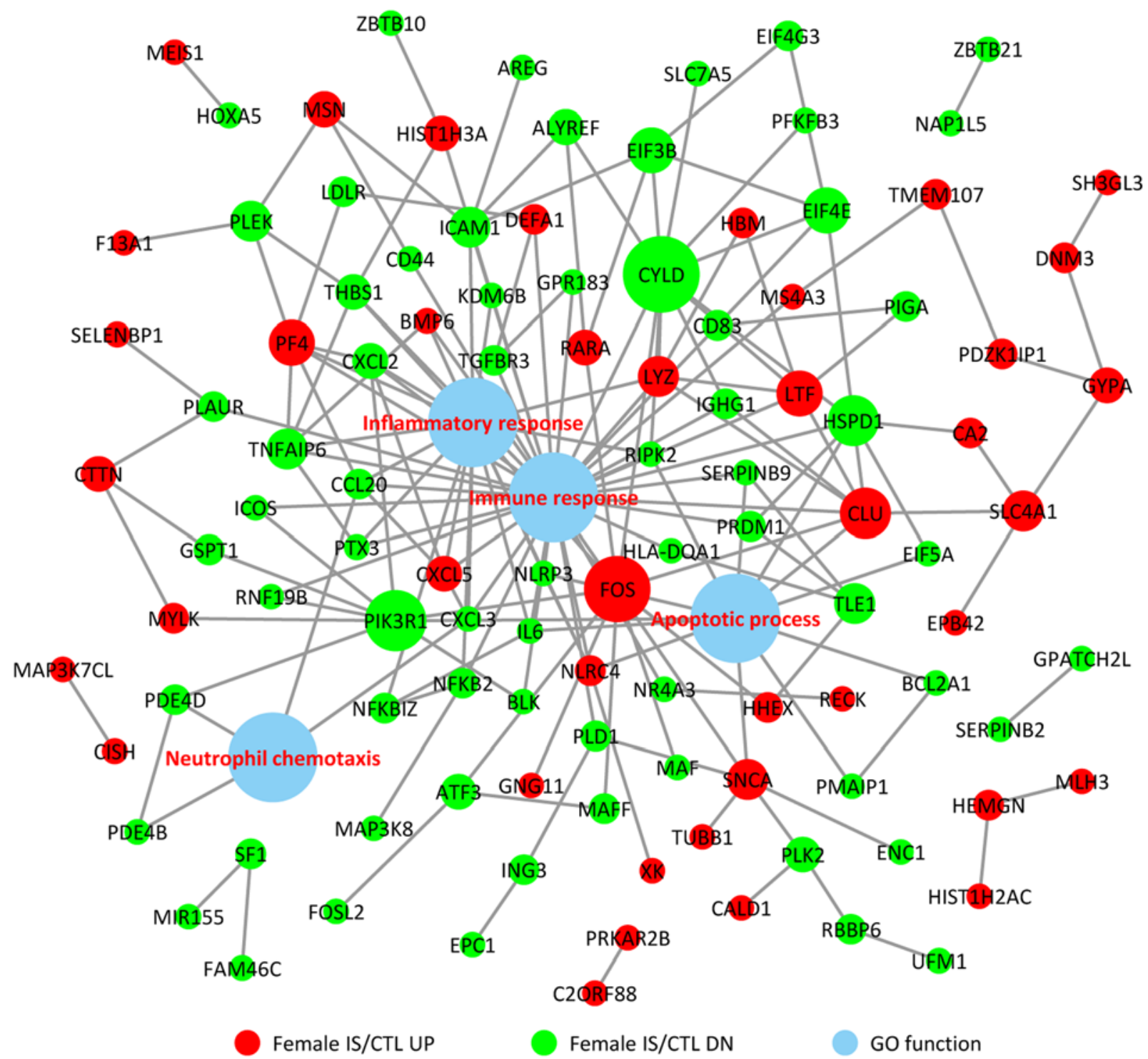

Figure 3. Protein-protein interaction of the upregulated and downregulated genes in female patients with IS and their association with immune response, inflammatory response, apoptotic process and neutrophil chemotaxis. CTL, control; DN, downregulated; GO, Gene Ontology; IS, ischemic stroke; UP, upregulated.

in the female IS group (solid red circles in Fig. 5); the downregulated target genes are also indicated, including TGFBR3, ETNK1, LDLR, RORA and RBBP6 (solid green circles in Fig. 5). The targets of miRNAs for the male IS/CTL group are shown in Fig. 6 (solid green circles represent downregulated target genes, such as RAB11FIP1, C5ORF51, GAB1 and SMAD5, and red circles represented upregulated target genes such as PPP1R15B, ELK4, MXD1 and LDLR).

Validation of DEGs in IS. Crosscheck validation analysis of female and male IS patient DEGs from GSE22255 and another set of Illumina HumanRef- 8 v3.0 expression beadchip (GSE16561) are shown in Fig. 7. There were 4 genes that are upregulated in both datasets in the female group including defensin $\alpha 1$ (DEFA1), pyruvate dehydrogenase kinase 4 (PDK4), caveolae associated protein 2 (SDPR) and transcobalamin 1 (TCN1) and only one shared upregulated gene in the male group, matrix metalloproteinase 9 (MMP9). These findings suggested that the differential expression targets selected had certain repeatability. Although numerous studies have been published regarding DEGs as candidate biomarkers for IS (35-37), the reliability of these findings remains uncertain, as they were generated from the studies without sufficient evidence of reproducibility and independent clinical validation.

\section{Discussion}

As a high-throughput genomics research technology, gene chips serve an important role in datamining of potential biological information and exploring the structure and function of the genome (38). Gene expression profiling experiments are mainly used to determine DEGs under different physiological or pathological conditions (39). Such analysis will facilitate the exploration of the relationship between health and disease at the molecular level and the potential mechanism through network regulation. However, the etiology and physiological pathology of IS are still not fully understood. Therefore, further clarifying the pathogenesis of IS, determining its risk 


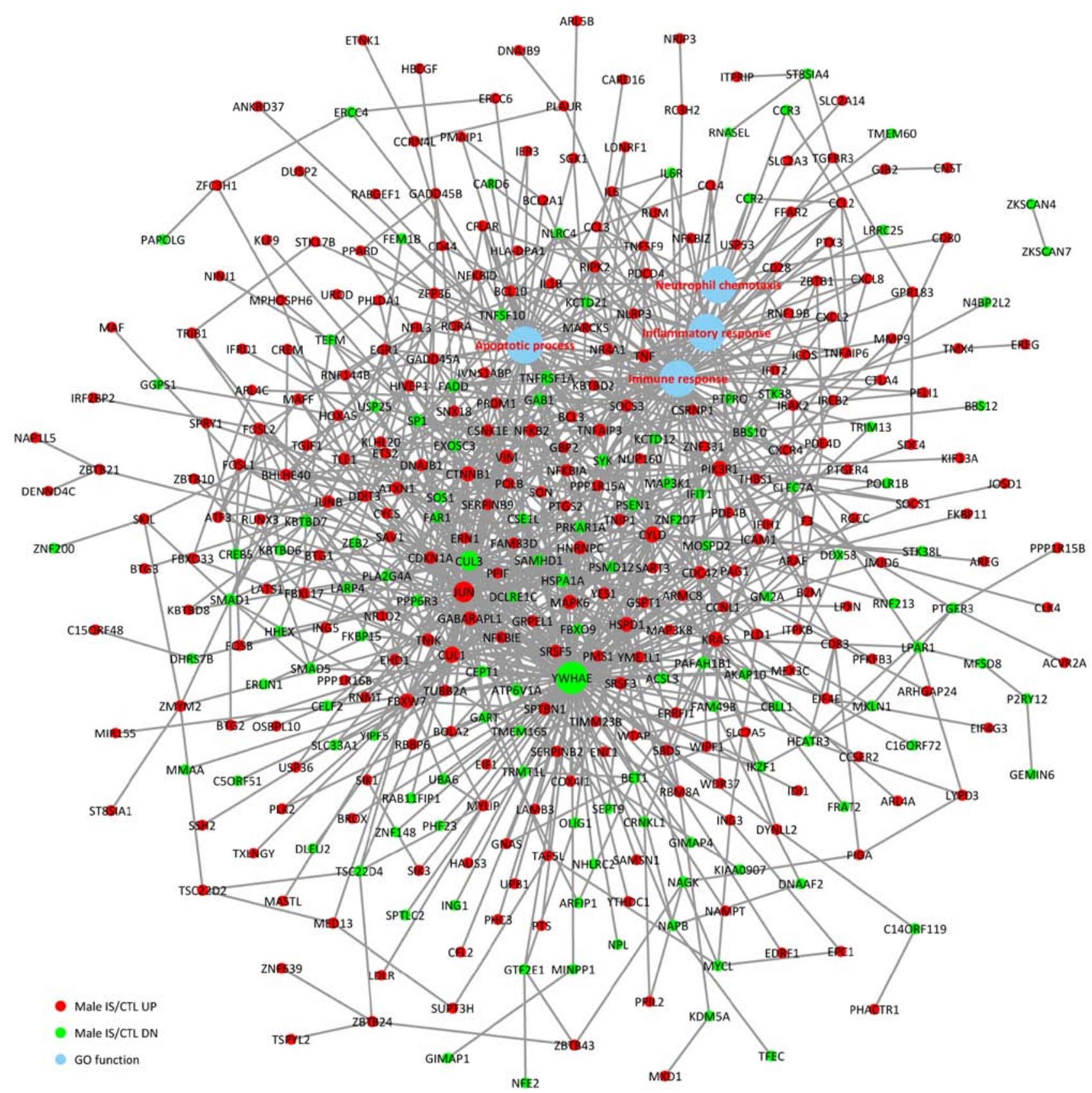

Figure 4. Protein-protein interaction of the upregulated and downregulated genes in male patients with IS and their association with immune response, inflammatory response, apoptotic process and neutrophil chemotaxis. CTL, control; DN, downregulated; GO, Gene Ontology; IS, ischemic stroke; UP, upregulated.

factors and developing effective therapeutic strategies will significantly improve the clinical outcome of stroke treatment.

The present study used bioinformatics tools and methods to screen the key DEGs and their functions in stroke. A total of 128 upregulated genes and 231 downregulated genes were found in female patients with IS, and 604 upregulated genes and 337 downregulated genes in male patients with IS. These DEGs were mainly associated with inflammatory response and apoptosis, and other biological pathways. Combining PPI and GO term enrichment results, it was also found that the genes involved in the GO analysis pathway were almost identical to the PPI network nodule-related genes, especially the CXCL family; tumor necrosis factor and PDK were most closely related to these pathways, which was consistent with the reported results.
Following a stroke, the damaged brain tissue releases proinflammatory chemokines, which trigger the inflammatory pathway in immune cells (40). Depending on the sequence of $\mathrm{N}$-terminal residues, chemokines can be divided into several different types, including CXC, CC, XC and CX3C, where C is an $\mathrm{N}$-terminal cysteine residue and $\mathrm{X}$ represents any amino acid residue (41). The chemokine ligands and receptors are emerging as potential therapeutic targets for cardiovascular diseases and have become increasingly studied in recent years. Brait et al (42) found that the expression of CXCR2 and its ligands CXCL1 and CXCL2 is significantly upregulated and peaks 1-3 days after injury in a middle cerebral artery occlusion (MCAO) rat model. Villa et al (43) inhibited CXCR1 with interleukin (IL)-8/CXCL8 receptor inhibitors in MCAO model rats and demonstrated that CXCR2, myeloperoxidase and 


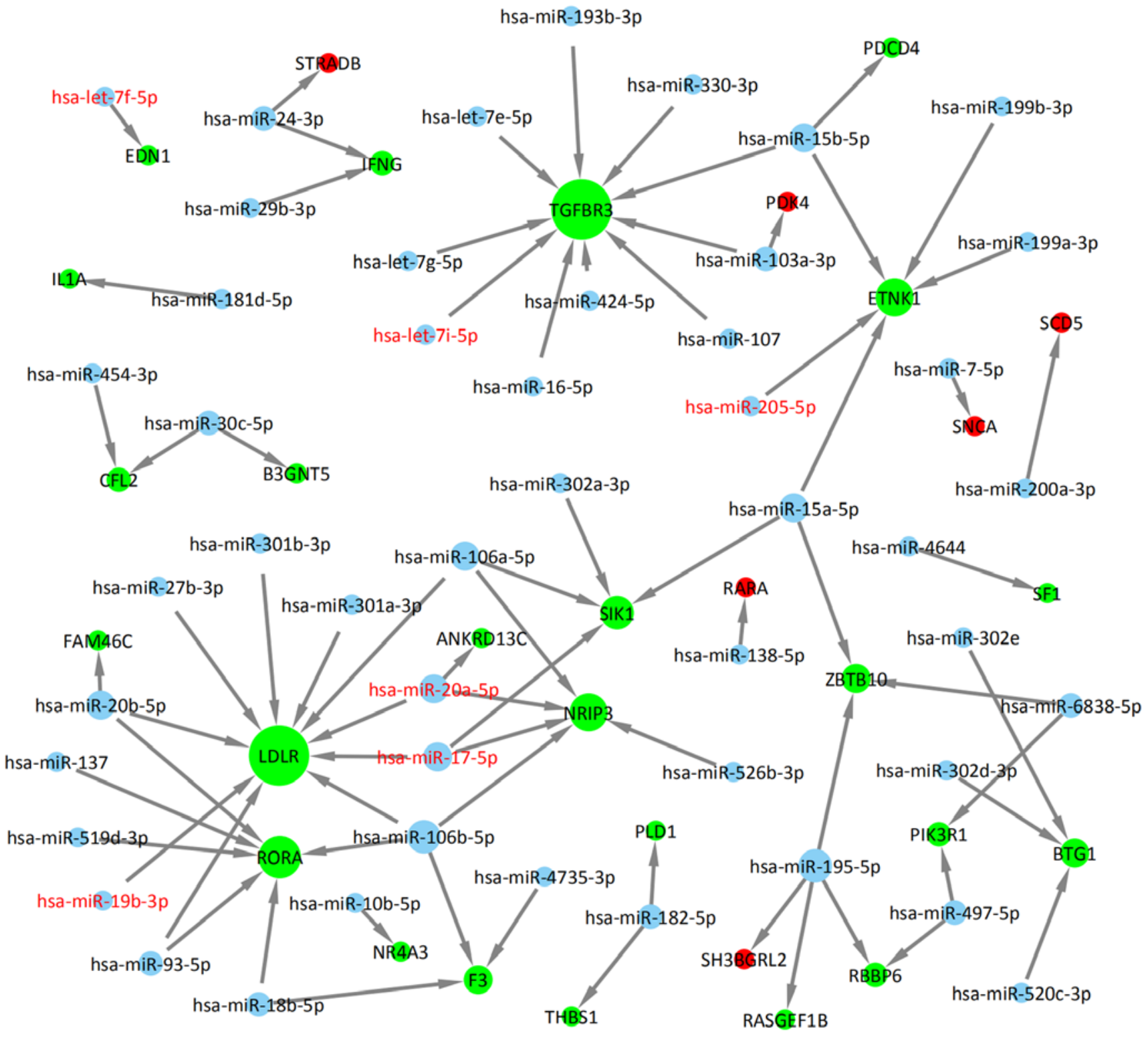

Female IS/CTL UP Female IS/CTL DN

Female IS/CTL DE miRNAs

Female other miRNAs

Figure 5. miRNA target network of the upregulated and downregulated genes of female patients with IS with differentially expressed miRNAs. CTL, control; diffexp, differentially expressed; DN, downregulated; IS, ischemic stroke; miRNA, microRNA; UP, upregulated.

IL-1A expression levels decreased, which reduced the symptoms of neurological deficits. In the present study, it was found that the chemokine receptors CXCL3, CXCL2 and CXCL8 were significantly upregulated in male patients with IS, and this was consistent with the previous reports.

PDK4 is a key enzyme that regulates pyruvate dehydrogenase complex (PDC) activity and is a key regulator of pyruvate oxidation and glucose maintenance in vivo (44). In calcified vascular smooth muscle cells and calcified vessels of patients with atherosclerosis, PDK4 expression is found to be upregulated alongside increased PDC phosphorylation, suggesting that PDK4 serves an important role in vascular calcification (45). In phosphate-treated vascular smooth muscle cells, PDK4 expression is upregulated, inducing mitochondrial dysfunction and, subsequently, apoptosis. Atherosclerosis is the result of cholesterol and lipid deposition on the arterial wall, often related to calcification, which is formed by the deposition of inorganic calcium on the arterial wall. Thus, the upregulation of PDK4 increased osteogenic markers and enhanced vascular calcification without significant effects on bone formation (46). This indicated that PDK4 may be a new target for the treatment of vascular calcification $(47,48)$.

In the pathogenesis of various injuries, the immune inflammatory signaling pathway caused by ischemia serves a major role, whereas ischemia itself serves a secondary role (49). In IS the key role of the immune inflammatory response has been widely recognized, but whether it plays a role in brain damage or brain protection may depend on the degree and stage of ischemia. Secondary injuries are caused by the inflammatory cascade following ischemia. Various inflammatory mediators, including cytokines, chemokines and cell adhesion molecules, aggravate ischemic brain damage $(27,28)$. In the early stage of ischemia, the inflammatory response exacerbates brain damage, and in the late 


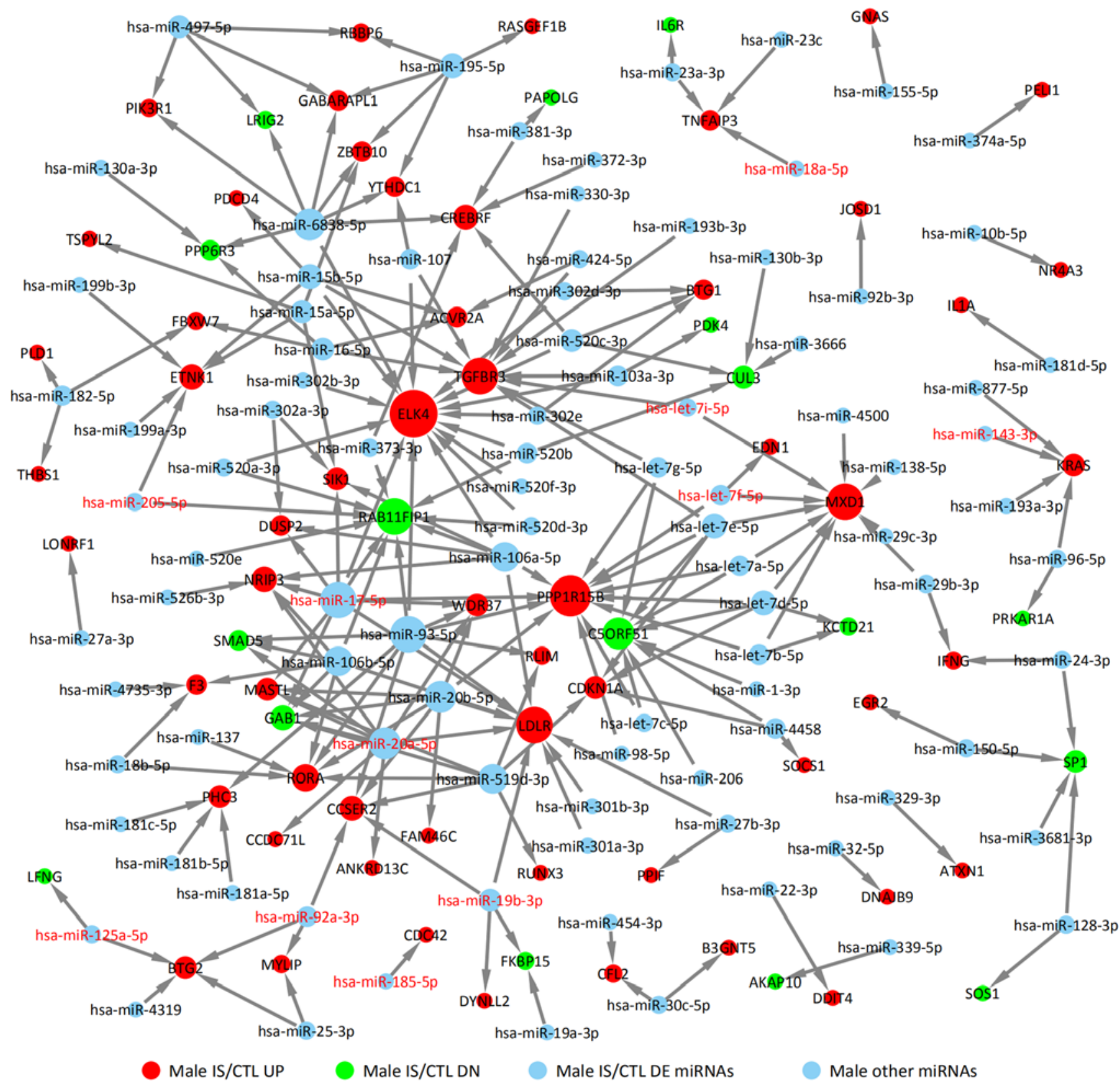

Figure 6. miRNA target network of the upregulated and downregulated genes of male patients with IS with differentially expressed miRNAs. CTL, control; diffexp, differentially expressed; DN, downregulated; IS, ischemic stroke; miRNA, microRNA; UP, upregulated.

stage of ischemia, it serves an important role in the recovery of nerve function and tissue repair. In addition, patients with IS are prone to a hypercoagulable state and advanced fibrinolysis. Most patients have coagulation changes in the clinic, which may lead to adverse events such as thrombosis and infarction, which affects the prognosis (50).

miRNAs are important post-transcriptional regulators that coordinate the regulation of target genes with multi-target mRNA. Risk factors involved in IS include hypertension, atherosclerosis, atrial fibrillation, diabetes and dyslipidemia. The role of miRNAs in the pathophysiology of stroke has become a focus of interest in recent research (51). A previous study reported that circulating miRNA expression in peripheral blood is relatively stable (52), suggesting that differential expression of specific miRNAs following stroke is expected to be a potential biomarker for diagnosis and prognosis, providing an effective theoretical basis for elucidating the pathogenesis of IS (53).

In the present study, the GSE110993 dataset was analyzed and 17 differentially expressed miRNAs (6 miRNAs in females and 11 miRNAs in males) were screened. According to previous studies, there are differences in the expression patterns of miRNAs in IS and in normal healthy people (54). For example, the abnormal expression of miR-221 is related to metabolic syndrome, whereas the expression of miR-221 in healthy people was significantly higher compared with expression levels in patients with IS (55). Studies have demonstrated that the abnormal expression level of miR-221 is significant for distinguishing healthy individual and those with cardiovascular and cerebrovascular diseases, tumor populations and endocrine populations $(56,57)$. Other previous studies have shown that miR-143/145 (57) and miR-125-5p (58) can also be used as 
Female
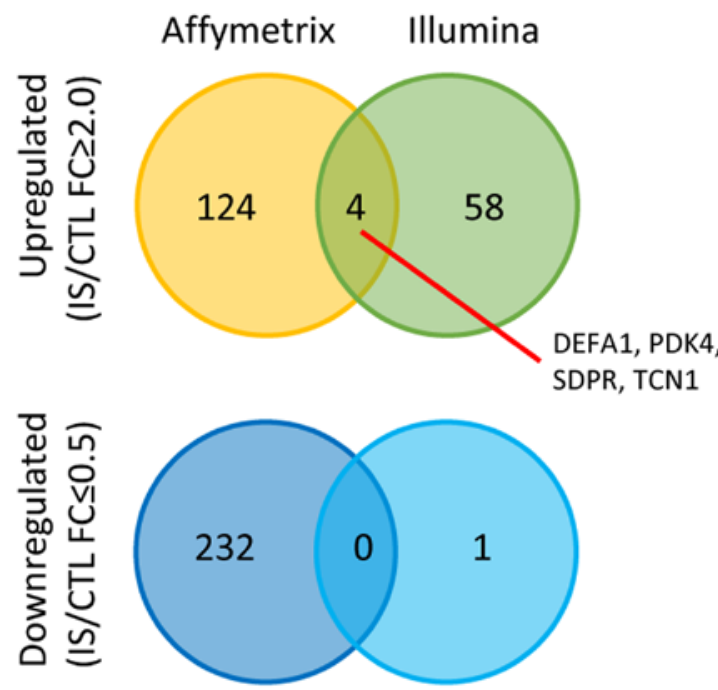

Male
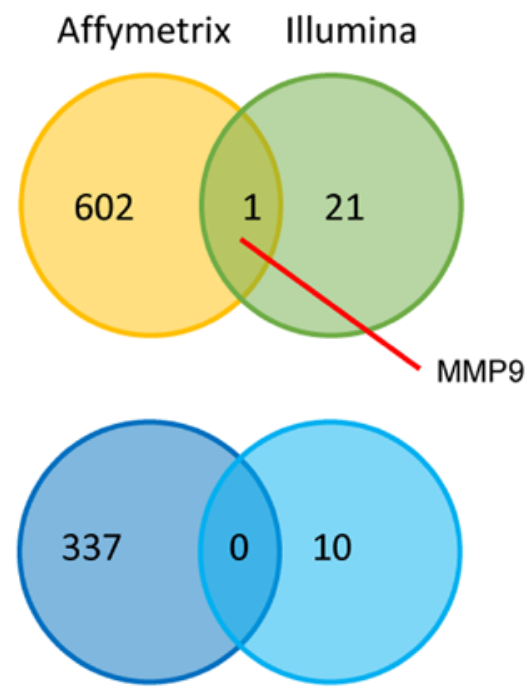

Figure 7. Overlapping upregulated and downregulated genes from two different beadchips (Affymetrix Human Genome U133 Plus 2.0 array and Illumina HumanRef-8 v3.0 expression beadchip) in female and male patients. CTL, control; FC, fold-change; IS, ischemic stroke; DEFA1, defensin alpha 1; PDK4, pyruvate dehydrogenase kinase 4; SDPR, caveolae associated protein 2; TCN1, transcobalamin 1; MMP9, matrix metalloproteinase 9.

markers for the risk of IS, which is consistent with the findings of the present study. miR-143/145 is most abundant in normal vascular tissues compared with other miRNAs and is selective in vascular smooth muscle cells expression. miR-125-5p has been found to be significantly upregulated in atherosclerosis (58). Inhibition of miR-125-5p expression significantly increases the uptake of lipids and enhances the clearance of human expression and secretion of inflammatory cell molecules (59). Some of these inflammatory cell molecules (60) may be involved in the inflammatory response of ox-LDL-stimulated monocytes, whereas ox-LDL may be taken up by macrophages, converted into foam cells that secrete pro-inflammatory factors and leads to cell necrosis and plaque formation. This series of chronic inflammatory reactions is atherosclerosis.

There are several limitations to the present study. First, it is notable that only one upregulated gene overlapped and none of downregulated genes overlapped between male and female groups. This may be due to differences in the effects of different types of sex hormones on platelet function. Androgens increase platelet activity, whereas estrogen inhibits its function (61). This may have an effect on the relative expression levels of mRNA and miRNA in the blood, suggesting that doctors might consider different medications for stroke patients of different sexes (62). Secondly, the data in Fig. 7 do not give confidence in the high-throughput genomics analyses because there are only 4 genes that are upregulated in both dataset in the female group and only one shared gene upregulated in the male group. This phenomenon may be because GSE22255 is based on GPL570 Affymetrix Human Genome U133 Plus 2.0 Array, whereas GSE16561 contained a total of 63 samples based on the platform of GPL6883 Illumina HumanRef- 8 v3.0 expression beadchip. On the one hand, these two different gene platforms contain different numbers and different types of genes; on the other hand, there may be detection errors in chips from Illumina and Affymetrix.

In summary, all of the miRNAs described above have a certain correlation with the pathogenesis and development of
IS, which is consistent with the findings of stroke researchers worldwide in recent years. The correlation between some miRNAs selected in this study have not been reported previously in the literature to the best of our knowledge. The possibility that these miRNAs are novel biomarkers for IS and its biological significance still needs further study. Further studies on the role of miRNAs will provide a theoretical basis for understanding the molecular mechanism of the pathogenesis and development of IS, as well as new research programs for prevention and treatment.

\section{Acknowledgements}

Not applicable.

\section{Funding}

This work was funded by HeBei Natural Science Foundations (grant no. H2017307016).

\section{Availability of data and materials}

The datasets used and/or analyzed during the current study are available from the corresponding author on reasonable request.

\section{Authors' contributions}

LL designed the experiments; $\mathrm{HW}$ and SS performed the experiments; LD and JC conducted the statistical analysis of the data and drafted the manuscript; $\mathrm{CZ}$ and $\mathrm{CB}$ conceived the study, participated in its design and coordination, and helped to draft the manuscript. All authors read and approved the final manuscript.

\section{Ethics approval and consent to participate}

Not applicable. 


\section{Patient consent for publication}

Not applicable.

\section{Competing interests}

The authors declare that they have no competing interests.

\section{References}

1. Ishak B, Abdul-Jabbar A, Singla A, Yilmaz E, von Glinski A Ramey WL, Blecher R, Tymchak Z, Oskouian R and Chapman JR: Intraoperative ischemic stroke in elective spine surgery: A retrospective study of incidence and risk. Spine (Phila Pa 1976) 45: 109-115, 2020.

2. Weiss HR, Mellender SJ, Kiss GK, Liu X and Chi OZ: Improvement in microregional oxygen supply/consumption balance and infarct size after cerebral ischemia-reperfusion with inhibition of p70 ribosomal S6 kinase (S6K1). J Stroke Cerebrovasc Dis 28: 104276, 2019.

3. Weisberg LA: 'Stroke and struck': Protecting the brain from cerebrovascular disease. South Med J 96: 331, 2003.

4. Singh RB, Suh IL, Singh VP, Chaithiraphan S, Laothavorn P, Sy RG, Babilonia NA, Rahman AR, Sheikh S, Tomlinson B and Sarraf-Zadigan N: Hypertension and stroke in Asia: Prevalence, control and strategies in developing countries for prevention. J Hum Hypertens 14: 749-763, 2000.

5. West R, Hill K, Hewison J, Knapp P and House A: Psychological disorders after stroke are an important influence on functional outcomes: A prospective cohort study. Stroke 41: 1723-1727, 2010

6. Ickenstein GW, Horn M, Schenkel J, Vatankhah B, Bogdahn U, Haberl R and Audebert HJ: The use of telemedicine in combination with a new stroke-code-box significantly increases t-PA use in rural communities. Neurocrit Care 3: 27-32, 2005.

7. Keller L, Hobohm C, Zeynalova S, Classen J and Baum P: Does treatment with t-PA increase the risk of developing epilepsy after stroke? J Neurol 262: 2364-2372, 2015.

8. Rudnick SI, Swaminathan J, Sumaroka M, Liebhaber S and Gewirtz AM: Effects of local mRNA structure on posttranscriptional gene silencing. Proc Natl Acad Sci USA 105: 13787-13792, 2008.

9. Park JH, Ahn JH, Song M, Kim H, Park CW, Park YE, Lee TK, Lee JC, Kim DW, Lee CH, et al: A 2-min transient ischemia confers cerebral ischemic tolerance in non-obese gerbils, but results in neuronal death in obese gerbils by increasing abnormal mTOR activation-mediated oxidative stress and neuroinflammation. Cell 8: 1126, 2019.

10. Liu S, Jin R, Xiao AY, Zhong W and Li G: Inhibition of CD147 improves oligodendrogenesis and promotes white matter integrity and functional recovery in mice after ischemic stroke. Brain Behav Immun 82: 13-24, 2019.

11. Feschotte C, Jiang N and Wessler SR: Plant transposable elements: Where genetics meets genomics. Nat Rev Genet 3 : 329-341, 2002

12. Sunkar R and Zhu JK: Novel and stress-regulated microRNAs and other small RNAs from Arabidopsis. Plant Cell 16: 2001-2019, 2004.

13. Cheng AM, Byrom MW, Shelton J and Ford LP: Antisense inhibition of human miRNAs and indications for an involvement of miRNA in cell growth and apoptosis. Nucleic Acids Res 33: 1290-1297, 2005.

14. Crippa S, Cassano M and Sampaolesi M: Role of miRNAs in muscle stem cell biology: Proliferation, differentiation and death. Curr Pharm Des 18: 1718-1729, 2012.

15. Kumar P, Wu H, McBride JL, Jung KE, Kim MH, Davidson BL, Lee SK, Shankar P and Manjunath N: Transvascular delivery of small interfering RNA to the central nervous system. Nature 448: 39-43, 2007.

16. Mehler MF and Mattick JS: Non-coding RNAs in the nervous system. J Physiol 575: 333-341, 2006.

17. Jung HL, Choi J, Hwang YK, Kim DH, Yoo KH, Sung KW and Koo HH: Inhibition of survivin expression by small interfering RNA (siRNA) in brain tumor cell lines. Cancer Res 65, 2005.

18. Pantano L, Friedländer MR, Escaramís G, Lizano E, Pallarès-Albanell J, Ferrer I, Estivill X and Martí E: Specific small-RNA signatures in the amygdala at premotor and motor stages of Parkinson's disease revealed by deep sequencing analysis. Bioinformatics 32: 673-681, 2016.
19. Roy J, Sarkar A, Parida S, Ghosh Z and Mallick B: Small RNA sequencing revealed dysregulated piRNAs in Alzheimer's disease and their probable role in pathogenesis. Mol Biosyst 13: 565-576, 2017.

20. Bam M, Yang X, Sen S, Zumbrun EE, Dennis L, Zhang J, Nagarkatti PS and Nagarkatti M: Characterization of dysregulated miRNA in peripheral blood mononuclear cells from ischemic stroke patients. Mol Neurobiol 55: 1419-1429, 2018.

21. Ma Q, Zhao H, Tao Z, Wang R, Liu P, Han Z, Ma S, Luo Y and Jia J: MicroRNA-181c exacerbates brain injury in acute ischemic stroke. Aging Dis 7: 705-714, 2016.

22. Watson SJ and Akil H: Gene chips and arrays revealed: A primer on their power and their uses. Biol Psychiatry 45: 533-543, 1999.

23. Johnston M: Gene chips: Array of hope for understanding gene regulation. Curr Biol 8: R171-R174, 1998.

24. Hochreiter S, Clevert DA and Obermayer K: A new summarization method for Affymetrix probe level data. Bioinformatics 22: 943-949, 2006

25. Teng L, Wang K, Liu Y, Ma Y, Chen W and Bi L: Based on integrated bioinformatics analysis identification of biomarkers in hepatocellular carcinoma patients from different regions. Biomed Res Int 2019: 1742341, 2019.

26. Gelaw YA, Williams G, Assefa Y, Asressie M and Soares Magalhães RJ: Sociodemographic profiling of tuberculosis hotspots in Ethiopia, 2014-2017. Trans R Soc Trop Med Hyg 113 379-391, 2019.

27. Thodberg M, Thieffry A, Vitting-Seerup K, Andersson R and Sandelin A: CAGEfightR: Analysis of 5'-end data using R/Bioconductor 20: 487, 2019.

28. Postma $\mathrm{M}$ and Goedhart J: PlotsOfData-A web app for visualizing data together with their summaries. PLoS Biol 17: e3000202, 2019.

29. Doncheva NT, Morris JH, Gorodkin J and Jensen LJ: Cytoscape String App: Network analysis and visualization of proteomics data. J Proteome Res 18: 623-632, 2019.

30. Scardoni G, Tosadori G, Faizan M, Spoto F, Fabbri F and Laudanna C: Biological network analysis with CentiScaPe: Centralities and experimental dataset integration. F1000Res 3: 139, 2014

31. Kalhori MR, Arefian E, Fallah Atanaki F, Kavousi K and Soleimani M: miR-548x and miR-4698 controlled cell proliferation by affecting the PI3K/AKT signaling pathway in Glioblastoma cell lines. Sci Rep 10: 1558, 2020.

32. Tang D, Zhao X, Zhang L and Wang C: Comprehensive analysis of pseudogene HSPB1P1 and its potential roles in hepatocellular carcinoma. J Cell Physiol, Jan 27, 2020 (Epub ahead of print).

33. Jiang W, Zheng L, Yan Q, Chen L and Wang X: miR-532-3p inhibits metastasis and proliferation of non-small cell lung cancer by targeting FOXP3. J BUON 24: 2287-2293, 2019.

34. Sticht C, De La Torre C, Parveen A and Gretz N: miRWalk: An online resource for prediction of microRNA binding sites. PLoS One 13: e0206239, 2018

35. Monbailliu T, Goossens J and Hachimi-Idrissi S: Blood protein biomarkers as diagnostic tool for ischemic stroke: A systematic review. Biomark Med 11: 503-512, 2017.

36. Li P, Teng F, Gao F, Zhang M, Wu J and Zhang C: Identification of circulating microRNAs as potential biomarkers for detecting acute ischemic stroke. Cell Mol Neurobiol 35: 433-447, 2015.

37. Dolmans LS, Rutten FH, Koenen NCT, Bartelink MEL, Reitsma JB, Kappelle LJ and Hoes AW: Candidate biomarkers for the diagnosis of transient ischemic attack: A systematic review. Cerebrovasc Dis 47: 207-216, 2019.

38. McColl BW, Rothwell NJ and Allan SM: Systemic inflammatory stimulus potentiates the acute phase and CXC chemokine responses to experimental stroke and exacerbates brain damage via interleukin-1- and neutrophil-dependent mechanisms. J Neurosci 27: 4403-4412, 2007.

39. Miller MC and Mayo KH: Chemokines from a structural perspective. Int J Mol Sci 18: pii: E2088, 2017.

40. Mirabelli-Badenier M, Braunersreuther V, Viviani GL, Dallegri F, Quercioli A, Veneselli E, Mach F and Montecucco F: $\mathrm{CC}$ and CXC chemokines are pivotal mediators of cerebral injury in ischaemic stroke. Thromb Haemost 105: 409-420, 2011.

41. Griffith JW, Sokol CL and Luster AD: Chemokines and chemokine receptors: Positioning cells for host defense and immunity. Annu Rev Immunol 32: 659-702, 2014.

42. Brait VH, Rivera J, Broughton BR, Lee S, Drummond GR and Sobey CG: Chemokine-related gene expression in the brain following ischemic stroke: No role for CXCR2 in outcome. Brain Res 1372: 169-179, 2011. 
43. Villa P, Triulzi S, Cavalieri B, Di Bitondo R, Bertini R, Barbera S, Bigini P, Mennini T, Gelosa P, Tremoli E, et al: The interleukin-8 (IL-8/CXCL8) receptor inhibitor reparixin improves neurological deficits and reduces long-term inflammation in permanent and transient cerebral ischemia in rats. Mol Med 13: 125-133, 2007.

44. Harris RA, Bowker-Kinley MM, Huang B and Wu PJ: Regulation of the activity of the pyruvate dehydrogenase complex. Adv Enzyme Regul 42: 249-259, 2002.

45. Holness MJ and Sugden MC: Regulation of pyruvate dehydrogenase complex activity by reversible phosphorylation. Biochem Soc Trans 31: 1143-1151, 2003.

46. Cohoon KP, Criqui MH, Budoff MJ, Lima JA, Blaha MJ, Decker PA, Durazo R, Liu K and Kramer H: Relationship of aortic wall distensibility to mitral and aortic valve calcification: The multi-ethnic study of atherosclerosis. Angiology 69: 443-448, 2018

47. Yang R, Zhu Y, Wang Y, Ma W, Han X, Wang X and Liu N: HIF-1 $\alpha /$ PDK4/autophagy pathway protects against advanced glycation end-products induced vascular smooth muscle cell calcification. Biochem Biophys Res Commun 517: 470-476, 2019.

48. Sugden MC and Holness MJ: Recent advances in mechanisms regulating glucose oxidation at the level of the pyruvate dehydrogenase complex by PDKs. Am J Physiol Endocrinol Metab 284: E855-E862, 2003.

49. Tobin MK, Bonds JA, Minshall RD, Pelligrino DA, Testai FD and Lazarov O: Neurogenesis and inflammation after ischemic stroke: What is known and where we go from here. J Cereb Blood Flow Metab 34: 1573-1584, 2014.

50. Cao Q, Wu J, Wang X and Song C: Noncoding RNAs in vascular aging. Oxid Med Cell Longev 2020: 7914957, 2020.

51. Gupta MK, Halley C, Duan ZH, Lappe J, Viterna J, Jana S, Augoff K, Mohan ML, Vasudevan NT, Na J, et al: miRNA-548c: A specific signature in circulating PBMCs from dilated cardiomyopathy patients. J Mol Cell Cardiol 62: 131-141, 2013.

52. Cho EY, Lee SJ, Nam KW, Shin J, Oh KB, Kim KH and Mar W: Amelioration of oxygen and glucose deprivation-induced neuronal death by chloroform fraction of bay leaves (Laurus nobilis). Biosci Biotechnol Biochem 74: 2029-2035, 2010.

53. He W, Chen S, Chen X, Li S and Chen W: Bioinformatic analysis of potential microRNAs in ischemic stroke. J Stroke Cerebrovasc Dis 25: 1753-1759, 2016.
54. Sørensen SS, Nygaard AB, Nielsen MY, Jensen K and Christensen T: miRNA expression profiles in cerebrospinal fluid and blood of patients with acute ischemic stroke. Transl Stroke Res 5: 711-718, 2014.

55. Huang D, Chen Z, Wang J, Chen Y, Liu D and Lin K MicroRNA-221 is a potential biomarker of myocardial hypertrophy and fibrosis in hypertrophic obstructive cardiomyopathy. Biosci Rep 40: pii: BSR20191234, 2020.

56. Di Martino MT, Arbitrio M, Fonsi M, Erratico CA, Scionti F, Caracciolo D, Tagliaferri P and Tassone P: Allometric scaling approaches for predicting human pharmacokinetic of a locked nucleic acid oligonucleotide targeting cancer-associated miR-221. Cancers (Basel) 12: pii: E27, 2019.

57. Wei YS, Xiang Y, Liao PH, Wang JL and Peng YF: An rs4705342 $\mathrm{T}>\mathrm{C}$ polymorphism in the promoter of miR-143/145 is associated with a decreased risk of ischemic stroke. Sci Rep 6: 34620, 2016.

58. Liang Y, Xu J, Wang Y, Tang JY, Yang SL, Xiang HG, Wu SX and $\mathrm{Li} \mathrm{XJ}$ : Inhibition of miRNA-125b decreases cerebral ischemia/reperfusion injury by targeting CK $2 \alpha / \mathrm{NADPH}$ oxidase signaling. Cell Physiol Biochem 45: 1818-1826, 2018.

59. Li JQ, Hu SY, Wang ZY, Lin J, Jian S, Dong YC, Wu XF, Lan D and Cao LJ: MicroRNA-125-5p targeted CXCL13: A potential biomarker associated with immune thrombocytopenia. Am J Transl Res 7: 772-780, 2015.

60. Chen T, Huang Z, Wang L, Wang Y, Wu F, Meng S and Wang C: MicroRNA-125a-5p partly regulates the inflammatory response, lipid uptake, and ORP9 expression in oxLDL-stimulated monocyte/macrophages. Cardiovasc Res 83: 131-139, 2009.

61. Shi C, Na N, Zhu X and Xu J: Estrogenic effect of ginsenoside Rg1 on APP processing in post-menopausal platelets. Platelets 24 51-62, 2013.

62. Giustino G, Overbey J, Taylor D, Ailawadi G, Kirkwood K, DeRose J, Gillinov MA, Dagenais F, Mayer ML, Moskowitz A et al: Sex-based differences in outcomes after mitral valve surgery for severe ischemic mitral regurgitation: From the cardiothoracic surgical trials network. JACC Heart Fail 7: 481-490, 2019.

This work is licensed under a Creative Commons Attribution-NonCommercial-NoDerivatives 4.0 International (CC BY-NC-ND 4.0) License. 